\title{
DoA Estimation in EM Lens Assisted Massive Antenna System Using Subsets Based Antenna Selection and High Resolution Algorithms
}

\author{
Sarmad Ahmed SHAIKH, Andrea M. TONELLO \\ Institute of Networked and Embedded Systems, Alpen-Adria-Universität Klagenfurt, Universitätsstraße 65-67, 9020 \\ Klagenfurt, Austria. \\ sashaikh@edu.aau.at, andrea.tonello@aau.at \\ Submitted July 12, 2017 / Accepted October 26, 2017
}

\begin{abstract}
In recent times, massive antenna array technology has captured significant attention among wireless communication researchers. This is a field with strong potential to increase rates of data transfer; mitigate interference and serve a large number of users simultaneously. To contribute further to this emerging technology, this paper presents an approach for the line-of-sight (LoS) based direction of arrival (DoA) estimation using the electromagnetic (EM) lens-focusing antenna concept. The EM lens focuses the received signal energy as a function of the angle of arrival (AoA) to a small subset/area of the antenna array. This is advantageous, as it helps to reduce both hardware implementation (RF chains) and the complexity of signal processing in the large number of antennas system. Furthermore, this focusing capability of the EM lens provides additional interference rejection gain which leads to estimate the DoA of user terminals precisely. Hence, in this work, subsets based antenna selection approach and subspace-based high resolution DoA estimation algorithms have been considered in combination with the EM lens assisted massive antenna system. In simulations where the DoA is estimated with the EM lens, the results are comparable with conventional methods of DoA estimation without an EM lens, despite the significantly reduced overall system complexity.
\end{abstract}

\section{Keywords}

Massive antenna system, electromagnetic (EM) lens, DoA estimation, wireless communication, 5G networks

\section{Introduction}

The massive antenna array system has recently captured the attention of the wireless research community over the conventional single-antenna and multiple-input-multiple-output (MIMO) system; and is becoming more attractive for next generation networks such as $5 \mathrm{G}$ [1]. The massive antenna or massive MIMO system consists of a very large number of antenna elements. It has been proposed in the literature to provide various advantages in spectral efficiency and link reliability. However, the large scale antenna array has also brought some challenges to be tackled in order to reap the benefits of this advanced technology. For the most part, the issues revolve around the large number of hardware (RF chains) implementations and the associated computational costs, which increase the complexity and the power consumption of the system [1-5]. In other words, if we have 100 antennas at the system's base station (BS) then we need to activate and deactivate $100 \mathrm{RF}$ chains simultaneously, where each RF chain includes a significant number of RF components such as amplifiers, mixers, analog to digital (A/D) and digital to analog (D/A) converters.

By taking into account these challenges, many solutions have been proposed in the relevant literature. For instance antenna selection and phase shifters as mentioned in [2], [4] where one has to compromise on the intended gains and/or the complexity of the large antenna array system. In [3], authors suggest using the dielectric lens enabled MIMO system for performance improvement and cost reduction. However, these techniques can be further investigated to address other important applications of the massive antenna systems such as the DoA estimation for the localization of user terminal. The DoA estimation having applications in efficient mobile communication (beamforming), radar, sonar, military, emergency services, autonomous driving and geographic routing; is an omnipresent task in array signal processing [6-10].

In the related literature, some novel works are reported about the direction finding in the antenna array processing systems. For example, in [12], [13], authors estimate the DoA of the received signals using the traditional method, the estimation of signal parameters via rotational invariance technique (ESPRIT), where all the antenna elements of the system are considered. However, this does not provide any advantage in minimizing the hardware complexity/costs. The dielectric cylindrical lens antenna architecture has been proposed in [14] for the angular estimation in the MIMO system. 


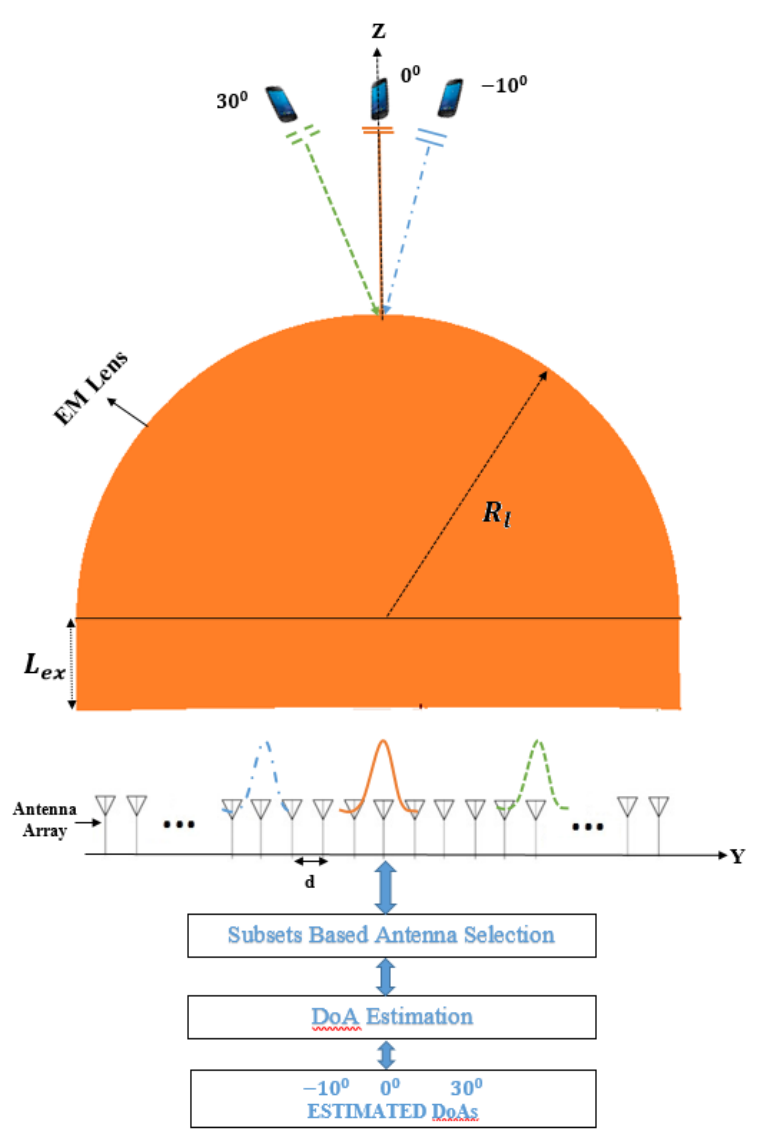

Fig. 1. Overview of the proposed design of the EM lens assisted massive antenna system.

However, this study has been conducted for the conventional MIMO system e.g, an antenna array with $N=4$ antenna elements, and requires the application of optimal power allocation schemes which raise the computational complexity. In addition to this, authors in [6] suggest using the Gaussian process regression method to detect the position through the fingerprints technique. Furthermore, in [6], [7], and the references therein authors indicate other direction finding methods such as the proximity-based localization (not free from complexities as it requires significant number of BSs), and the localization based on the joint estimation of delay, angle of departure (AoD), and AoA. Although these techniques can provide the desired results, they involve a significant amount of hardware and computational costs.

Thus, in order to achieve the promising advantages of the large number of antenna system and yet reduce its complexity, this paper presents a DoA estimation approach using the EM lens-focusing antennas concept as shown in Fig. 1, where $R_{1}$ and $L_{\text {ex }}$ represent the radius and extension length of a typical extended hemisphere lens, respectively. Thanks to the focusing property of the EM lens, the proposed approach does not only reduce the complexity of the hardware and signal processing but also it rejects the interference of other incoming signals by exciting subsets of antennas as a function of the AoA. Hence, this study (extending our basic idea in [5], [8]) provides a detailed investigation of the LoS-DoA estimation in the EM lens assisted massive antenna system. By proposing an EM lens-focusing subsets based antenna selection technique followed by subspace-based high resolution DoA estimation algorithms such as the multiple signal classification (MUSIC), ESPRIT and Root-MUSIC, we investigate the performance and/or complexity reduction achievable. As a result, these insights will become helpful for designing a practical DoA estimation system with application in future wireless communication networks such as $5 \mathrm{G}$.

The rest of the paper is organized in the following manner. Section 2 presents the system model for the EM lens assisted massive antenna system. Section 3 explains the EM lens focused subsets based antenna selection and the DoA estimation process. The simulation results observed and the parameters assumed, are provided in Sec. 4. Finally, we conclude the paper in Sec. 5 with future work directions.

\section{System Model}

Generally, the antenna array consists of a number of antenna elements and is designed to produce the desired radiation pattern [15-18]. Moreover, it provides significant advantages in estimating the DoA of received signals and maximizing the signal-to-interference-plus-noise ratio (SINR) [16], as mentioned earlier. This is why, typically, the massive antenna system considers numerous antenna elements in its design configuration in order to enhance the performance to a significant level. The array configuration can be linear (most elementary), circular, planar or conformal. The uniform linear array (ULA) is the most simple and widely used array design in practice. Therefore, for simplicity, we assume a ULA in this study.

The proposed approach for DoA estimation in the massive antenna system considers finding the AoA of the received signal. In this regard, we propose to integrate the EM lens with the ULA of the massive antenna elements at the BS and call this setup the EM lens-assisted massive antenna system. We combine the EM lens with ULA because the EM lens provides the advantage of varying the path of incident EM waves in the desired direction. This property of the lens makes it possible to excite/focus the received signal passing through the lens on a particular antenna subset (consisting of a small number of antennas) on the ULA as a function of the AoA of the received signal. Consequently, it contributes to reduce the number of antennas to be processed and the associated computational costs because we can now instantaneously select and process fewer focused antennas while deactivating the unfocused antennas. Additionally, as mentioned in remark 2-3 of [3], this ability of the EM lens to focus the received signal energy on different subset of antennas as a function of the angle of arrival (AoA) of the received signal helps to reduce the interference from other incoming signals. This is in sharp contrast to the traditional massive MIMO system without EM lens because in the conventional system without EM lens, on average, the power received fromeach user signal is evenly distributed across all the antennas. 


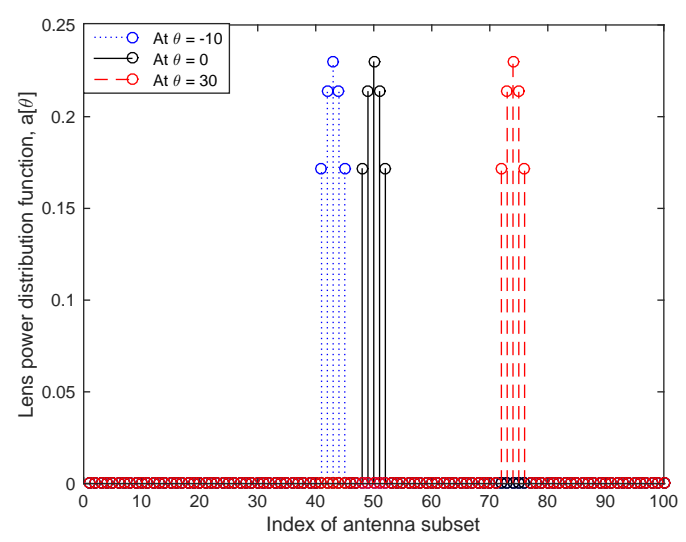

Fig. 2. Power distribution on the antenna subsets (at $\Delta=2$ or correspondingly $J=5$ ) focused by the lens as a function of the incident angle.

Therefore, for each user, the desired signals at all the $M$ receive antennas are equally corrupted by the other user signals. Thus, by utilizing the focusing property of the EM lens assisted massive antenna system, we can efficiently estimate the DoA of the received signals by applying one of the DoA estimation algorithms in the focused antenna subsets of the ULA. In this study, we consider the subspace-based high resolution DoA estimation algorithms in combination with the lens antennas system.

In order to get more information about the EM lens, we recommend reading the article in [11], [19] and the references therein. However, it is necessary to discuss the formation of the subset of antenna elements on the ULA at the BS. The signal that is focused on a subset of antenna elements by the EM lens, is actually a function of the AoA of the incident signal. Depending on the geometric design parameter $\Delta$ of the EM lens, the size of the focused subset of $J$ antennas is generally calculated as $J=2 \Delta+1 \ll M$, where $M$ represents the total number of antenna elements deployed in the ULA at the BS. Moreover, the desired size EM lens can be designed using the dielectric material (i.e., silicon, and quartz) with surfaces curved from front and/or rear. Furthermore, the spatial power distribution of the arriving signal passing through the EM lens and focused on a subset is a function of the AoA of the incident EM wave. In this regard, Fig. 2 shows intuitively that as the received signal's incident angle $\theta$ varies, the focused power distribution on the subset (peak-location) sweeps accordingly. Note that in this work we assume that the antenna array at the BS is centered at zero and placed in the vertical $y$-axis. For simplicity, in this work we consider a single-cell uplink LoS transmission with angle coverage range of the array from -60 to +60 degrees.

Having outlined this essential information, we now describe the channel model for the massive antenna array system without lens and with lens in the following subsections.

\subsection{Channel Model of the System without Lens}

Let us consider $N$ single-antenna user terminals that transmit the signals towards a BS consisting of an $M$-elements ULA. Furthermore, the transmitted $n^{\text {th }}$ user's $(n \in \mathrm{N})$ narrowband signal impinges as a plane wave on the ULA with the AoA, $\theta_{n}$. In this way, the channel coefficient $h_{n m}$ between the $n^{\text {th }}$ user terminal and the $m^{\text {th }}$ antenna element can be defined as

$$
h_{n m}=\sqrt{\alpha_{n}} \times \exp \left(\mathrm{j} \psi_{n}+\mathrm{j} \frac{2 \pi d}{\lambda}(m-1) \sin \theta_{n}\right)
$$

where $\alpha_{n}$ represents the channel gain of user $n$ which is determined by the distance-dependent signal attenuation and shadowing. $\psi_{n}$ is the phase shift of user terminal $n$ and can be modeled as a random variable with the uniform distribution over $[0,2 \pi] . \lambda$ denotes the free space wavelength and $d$ shows the inter-antenna elements spacing in the ULA. Furthermore, $\theta_{n} \in[-\phi, \phi]$ is the AoA of the $n^{\text {th }}$ user where $\phi \in(0, \pi]$ represents the range of angle coverage of the antenna array. Thus, we can define the covariance matrix, required by the DoA algorithm, as $\mathbf{R}_{n}=\mathbb{E}\left[\mathbf{h}_{n} \mathbf{h}_{n}^{\mathrm{H}}\right]$ where $\mathbf{h}_{n}=\left[h_{n 1}, h_{n 2}, h_{n 3}, \ldots, h_{n M}\right]^{\mathrm{T}}$ is the channel vector of user $n$. Further, $\left[\mathbf{R}_{n}\right]_{m k}=\mathbb{E}\left[h_{n m} h_{n k}^{*}\right]$ provides the $(m, k)$-th entry of the covariance matrix $\mathbf{R}_{n}$.

\subsection{Channel Model of the System with Lens}

Now, we consider the EM lens in the system as shown in Fig. 1. In this case, by integrating the lens with the ULA we can obtain the channel coefficient equation as [5], [19]

$$
\hat{h}_{n m}=\sqrt{\alpha_{n}} \sqrt{a_{m}\left(\theta_{n}\right)} \times \exp \left(\mathrm{j} \psi_{n}+\mathrm{j} \frac{2 \pi d}{\lambda}(m-1) \sin \theta_{n}\right)
$$

where all parameters are same as those defined earlier except $a_{m}\left(\theta_{n}\right)$. This additional factor $a\left(\theta_{n}\right)$ is the power distribution on the subset of $J$ antenna elements focused by the EM lens as a function of the AoA of the $n^{\text {th }}$ user, with $a_{m}\left(\theta_{n}\right) / M$ reflecting the power fraction obtained by the $m^{\text {th }}$ antenna element. The factor $a_{m}\left(\theta_{n}\right)$ depending on design parameter $\Delta$, can be defined by using a power density function i.e., $f(i ; \theta)$ with considering mean $\bar{i}(\theta)$ and variance $\sigma^{2}$, as

$$
a_{m}(\theta)= \begin{cases}c \int_{i_{m}-d / 2}^{i_{m}+d / 2} f(i ; \theta) \mathrm{d} i, & \left|m-m^{*}(\theta)\right| \leq \Delta, \\ 0, & \text { otherwise }\end{cases}
$$

where $c$ is a constant such that $\sum_{m=1}^{M} a_{m}(\theta)=M, i_{m}$ denotes the $m^{\text {th }}$ antenna location (in terms of $\lambda$ ) at the BS and $m^{*}(\theta)$ is the peak power location that is based on the AoA of the excited subset. As mentioned in [20], $f(i ; \theta)$ can be modeled by a Gaussian power distribution function with mean and variance as indicated earlier.

Similarly, let us define $\hat{\mathbf{h}}_{n}=\left[\hat{h}_{n 1}, \hat{h}_{n 2}, \hat{h}_{n 3}, \ldots, \hat{h}_{n M}\right]^{\mathrm{T}}$ as the channel vector of user $n$ with the EM lens; then the covariance matrix can be formed as $\hat{\mathbf{R}}_{n}=\mathbb{E}\left[\hat{\mathbf{h}}_{n} \hat{\mathbf{h}}_{n}^{\mathrm{H}}\right]$. Hence, it can be seen that the effective channel covariance matrix of any particular user terminal changes as the power distribution function $a(\theta)$ changes [3]. However, the total received signal energy of user $n$ captured by the subset of antennas remains same as with - or without- the EM lens because the EM lens 
only focuses the power distribution of the incident signal on the subset of the ULA. In this way, we can define the received signal model as follows.

Consider a ULA with $M$ identical antenna elements spaced by distance $d$. Furthermore, by considering the antenna element 1 as the reference element, the array receives the far field signals, impinging the array with the angle of incidence $\theta$. The received signal of user $n$ at $m^{\text {th }}$ element is delayed by $\exp \left(\frac{(m-1) d \sin }{\mathrm{c}}\right)$ as compared to the signal $z_{n 1}(t)=\sqrt{\alpha_{n}} \sqrt{a_{1}\left(\theta_{n}\right)} s_{n}(t)$ received at the first/origin antenna element [21], where $s$ is the actual data symbol of user $n$ and $\mathrm{c}$ is the speed of light. Thus, at element $m$, the $n^{\text {th }}$ user's received signal is given by

$$
\begin{aligned}
z_{n m}(t)= & \sqrt{\alpha_{n}} \sqrt{a_{m}\left(\theta_{n}\right)} \\
& \times \exp \left(\mathrm{j} \frac{2 \pi d(m-1) \sin \left(\theta_{n}\right)}{\lambda}+\mathrm{j} \psi_{n}\right) s_{n}(t)+w(t) \\
& =\hat{h}_{n m} s_{n}(t)+w(t)
\end{aligned}
$$

where $w$ is the noise. Moreover, in general, the signal received by $M$-antenna elements array (without EM lens case) with $\psi=0$ can be defined as

$$
\begin{aligned}
\mathbf{z}(t)=\left[\begin{array}{c}
z_{1}(t) \\
z_{2}(t) \\
z_{3}(t) \\
\vdots \\
z_{M}(t)
\end{array}\right] & =\left[\begin{array}{c}
1 \\
\mathrm{e}^{-\mathrm{j} k d \sin (\theta)} \\
\mathrm{e}^{-\mathrm{j} 2 k d \sin (\theta)} \\
\vdots \\
\mathrm{e}^{-\mathrm{j}(M-1) k d \sin (\theta)}
\end{array}\right] s(t)+w(t) \\
& =\mathbf{v}(\theta) s(t)+\mathbf{w}(t)
\end{aligned}
$$

where, $\mathbf{v}(\theta)$ is the steering vector. Now, if we consider $N$ number of signal sources, transmitting the signals from directions $\left(\theta_{1}, \theta_{2}, \ldots, \theta_{N}\right)$, then the signal model can be represented as

$$
\begin{gathered}
\mathbf{Z}(t)=\sum_{n=1}^{N} \mathbf{v}\left(\theta_{n}\right) s_{n}(t)+\mathbf{w}(t) \\
\left.\mathbf{Z}(t)=\left[\begin{array}{c}
s_{1}(t) \\
s_{2}(t) \\
\vdots \\
s_{N}(t)
\end{array}\right]+\mathbf{v}\left(\theta_{2}\right), \ldots, \mathbf{v}\left(\theta_{N}\right)\right]=\mathbf{V} \mathbf{s}(t)+\mathbf{w}(t)
\end{gathered}
$$

where $\mathbf{V}$ is the array steering matrix for $N$ users and is given by

$$
\mathbf{V}=\left[\begin{array}{cccc}
1 & 1 & \ldots & 1 \\
\mathrm{e}^{-\mathrm{j} k d \sin \left(\theta_{1}\right)} & \mathrm{e}^{-\mathrm{j} k d \sin \left(\theta_{2}\right)} & \ldots & \mathrm{e}^{-\mathrm{j} k d \sin \left(\theta_{N}\right)} \\
\mathrm{e}^{-\mathrm{j} 2 k d \sin \left(\theta_{1}\right)} & \mathrm{e}^{-\mathrm{j} 2 k d \sin \left(\theta_{2}\right)} & \ldots & \mathrm{e}^{-\mathrm{j} 2 k d \sin \left(\theta_{N}\right)} \\
\vdots & \vdots & \ddots & \vdots \\
\mathrm{e}^{-\mathrm{j}(M-1) k d \sin \left(\theta_{1}\right)} & \mathrm{e}^{-\mathrm{j}(M-1) k d \sin \left(\theta_{2}\right)} & \ldots & \mathrm{e}^{-\mathrm{j}(M-1) k d \sin \left(\theta_{N}\right)}
\end{array}\right]
$$

However, in the EM lens case, using (2), $\mathbf{V}$ can be defined as

$$
\hat{\mathbf{V}}=\left[\begin{array}{cccc}
\hat{h}_{1, m_{1}} & \hat{h}_{2, m_{1}} & \ldots & \hat{h}_{N, m_{1}} \\
\hat{h}_{1, m_{2}} & \hat{h}_{2, m_{2}} & \ldots & \hat{h}_{N, m_{2}} \\
\vdots & \vdots & \ddots & \vdots \\
\hat{h}_{1, m_{J}} & \hat{h}_{2, m_{J}} & \ldots & \hat{h}_{N, m_{J}}
\end{array}\right]=\left[\hat{\mathbf{v}}\left(\theta_{1}\right) \hat{\mathbf{v}}\left(\theta_{2}\right), \ldots, \hat{\mathbf{v}}\left(\theta_{N}\right)\right]
$$

where subscript $m_{1} \ldots m_{J}$ denotes the $J$ indexes of the excited antennas of a particular $n^{\text {th }}$ user or subset. Hence, for $n^{\text {th }}$ user, $\hat{\mathbf{z}}_{n}(t)$ signal is observed at the BS and is given as

$$
\hat{\mathbf{z}}_{n}(t)=\hat{\mathbf{v}}\left(\theta_{n}\right) s_{n}(t)+\mathbf{w}(t)
$$

In this way, for $N$ different incident angles $\left[\theta_{1}, \theta_{2}, \ldots, \theta_{N}\right]$ excited subsets, we have $N$ received signals, such that

$$
\hat{\mathbf{Z}}(t)=\left[\hat{\mathbf{z}}_{1}(t), \hat{\mathbf{z}}_{2}(t), \ldots, \hat{\mathbf{z}}_{N}(t)\right]
$$

\section{Antenna Selection and DoA Estima- tion}

Once the EM lens-focusing antenna system is designed properly with a particular design parameter $\Delta$ as discussed in Sec. 2, the process of selecting the excited subsets and the estimation of the DoA can be followed. In this regard, let us consider a vector $\mathbf{y}=[0,0, \ldots, 0]_{1 \times M}$ of length $M$ with zero entry values, where each element of $\mathbf{y}$ corresponds to an antenna element of the ULA. In this way, according to our proposed method, whenever any signal arrives from a particular direction, it is focused by the EM lens on a subset of $J$ antenna elements; where $J=2 \Delta+1$, as mentioned previously. Thus, for $N$ user signals, at most $N \times J$ entries of $\mathbf{y}$ can be nonzero at one time and distributed according to the expression in (3) and shown in Fig. 3. It is worth mentioning here that in each excited subset, the central antenna element can capture the maximum signal energy compared to the rest of the focused elements in that excited subset. Moreover, this process can be visualized in Fig. 4 where after forming the subsets or clusters (each consists of few closely spaced subsets), nonzero data entries of $\mathbf{y}$ can be extracted and supplied to the DoA estimation block to find the DoA of the focused signals. In this regard, many DoA estimation algorithms

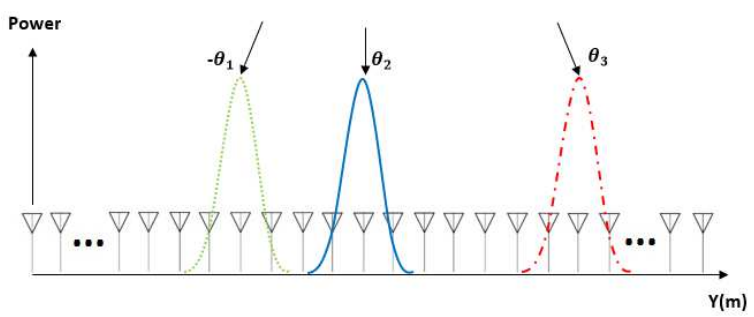

Fig. 3. Signal power distribution in the excited subsets on the ULA (at $\Delta=1$ ).

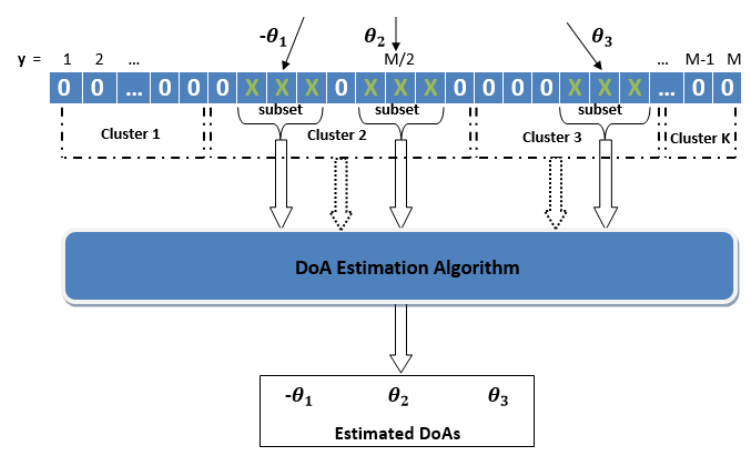

Fig. 4. Process for excited subsets/clusters selection and DoA estimation (at $\Delta=1$ ). 
exist in literature such as the Capon's method, Bartlett method, minimum variance distortionless response (MVDR), min-norm, classical beamformer, MUSIC, ESPRIT and Root-MUSIC [8], [9], [22-25]. However, as mentioned earlier, we will discuss and apply the subspace-based MUSIC, ESPRIT and Root-MUSIC high resolution algorithms in combination with the EM lens assisted massive antenna system. In following subsections we give details about this procedure.

\subsection{Subsets Based Antenna Selection}

From the previous discussion, it can be noted that the EM lens provides the advantage of focusing the received signal on a small subset of antenna elements so that we can process fewer antenna elements per user signal. Nevertheless, it typically requires activation of all $M$ antenna elements and corresponding $M$-RF chains. Consequently, it increases the cost of the hardware (RF chains) implementation and power consumption. Hence the antenna selection (AS) becomes necessary to select $S$ optimal antennas out of $M$ for the processing of the received signals. As a result, AS helps to reduce the RF chains from $M$ to $S$ [26] where $S \ll M$. Generally, the optimal AS algorithm requires instantaneous CSI for all the BS antennas $M$ which leads to raising the training time by a factor of $M / S$ [26]. This may result in significant performance degradation due to less time available for data sending. Few other AS schemes are mentioned in [27], [28] where the phase shifters/RF switches network in RF-precoding block and channel capacity equation are utilized to perform the optimal AS process. However, in order to select the best $S$ out of $M$ antennas/RF-chains, most of the AS schemes must perform an exhaustive search over $\left(\begin{array}{c}M \\ S\end{array}\right)$ possible options. This may increase the order of complexity for the large $M$ and the moderate $S$. Therefore, here we propose an antenna selection approach based on peak power location search in the $M$ antennas array.

In the EM lens-enabled massive antenna system, the power of the received signal is distributed unevenly (i.e. bell shape) in the excited subset's antennas as mentioned earlier and shown in Fig. 3. In this case, subset's central antenna element (peak power location) or a few antennas closer to the central element can receive the maximum signal energy than the other antennas in the subset. This power distribution property of the EM lens assisted system provides the opportunity to select $J=2 \Delta+1 \subset S$ excited antennas subset out of $M$, based on searching the maximum (peak power locations) energies across the antenna array with a threshold of $\gamma$. In this regard, once the peak power location $m^{*}$ is identified, we can find other subset antennas by selecting the $m^{*}+\Delta$ and $m^{*}-\Delta$ elements. Moreover, by using RF switches we can process $S$ antennas from all excited subsets. However, the scheme can be extended to further reduce the RF-chains if only the antennas which are closer to the peak power location element $m^{*}$ in each corresponding subset are selected. This is because the antennas which are farther away from $m^{*}$ receive much less energy and hence their contribution could be negligible; especially for the DoA estimation process.

\subsection{DoA Estimation}

In order to estimate the DoA of the excited subsets, the selected antennas' signals are advanced to the DoA estimation algorithm which can be applied in two ways. We can either apply the DoA algorithm individually in each selected subset's signal; or in each determined cluster's signals, where a cluster consists of a group of the closely spaced subsets which are optimally selected according to our previous discussion. The former method requires applying the DoA estimation algorithm an equal number of times as the total number of the focused subsets. This may increase the iterations of the DoA algorithm. However, in the latter case, as shown in Fig. 4, we need to apply the DoA algorithm up to the total number of clusters determined. As a result, this helps to minimize the number of operational efforts of applying the DoA algorithm.

By applying these two techniques and using the MUSIC algorithm (explained in the subsection below), the estimated DoAs with lens versus without lens, are provided in Fig. 5 and Fig. 6, respectively. It can be observed that, for the both techniques (individual and clustering subsets consideration) the estimated spectrum peaks of the proposed method are comparable to the without EM lens case. Moreover, with approximately the same results as obtainable from each selected individual subset, the number of times the DoA algorithm must be operated can be minimized further by determining a cluster over the closely spaced subsets. Here, for all the examples, we assume $M=100, \Delta=3, \mathrm{SNR}=10 \mathrm{~dB}$ and snapshots of a signal $=200$ unless stated differently. With this information, we now briefly explain the subspace-based high resolution DoA estimation algorithms and give example for each algorithm by considering with lens and without lens scenarios.

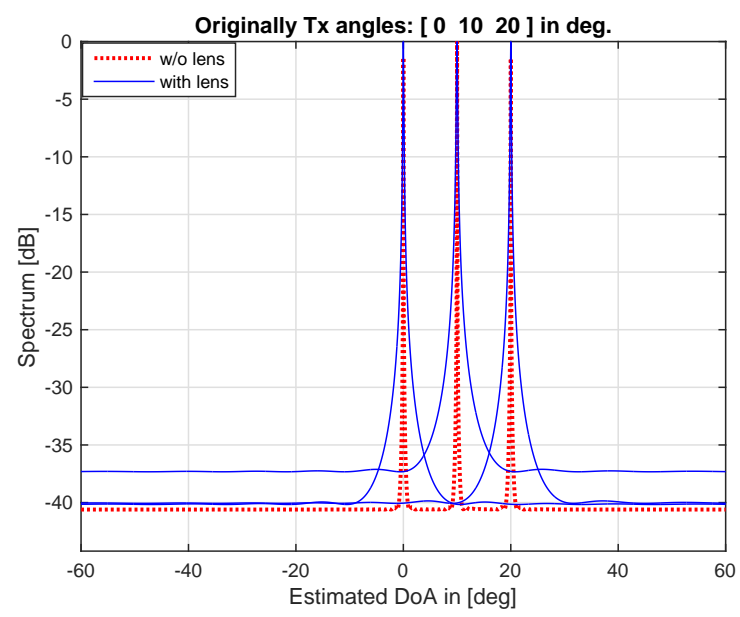

Fig. 5. Estimated DoAs with lens (individual excited subset) versus without lens. 


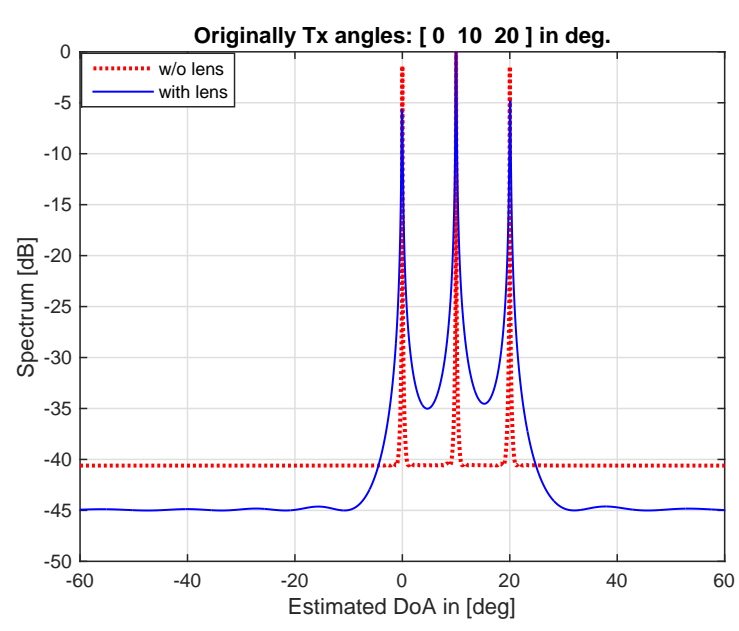

Fig. 6. Estimated DoAs with lens (a particular determined cluster over a group of the closely spaced excited subsets) versus without lens.

\subsubsection{MUSIC Algorithm}

This technique performs the eigenvalue decomposition (EVD) of the covariance matrix of the received signal on a group of antenna elements, in order to form the EVD subspace. Later, it is split into two parts, $M \times N$ signal subspace $\left(E_{S}\right)$ and the $M \times(M-N)$ noise subspace $\left(E_{N}\right)[21,25,29]$. In this way, we can estimate the DoA of the received signals by using the MUSIC pseudospectrum that is given as

$$
\mathbf{p}_{\text {MUSIC }}(\theta)=\frac{1}{\left|\mathbf{v}^{H}(\theta) \mathbf{E}_{N} \mathbf{E}_{N}^{H} \mathbf{v}(\theta)\right|} .
$$

Due to the reciprocal of this matrix product in (12), sharp peaks are created at the AoA axis on the plot showing the estimated DoAs. Note that by using EM-lens, the dimensions of the covariance and the corresponding EVD matrices are reduced from $\boldsymbol{M} \times \boldsymbol{M}$ to $\boldsymbol{J} \times \boldsymbol{J}$. Consequently, the DoA can be efficiently estimated with signal processing complexity in the order of $J$ and not $M$.

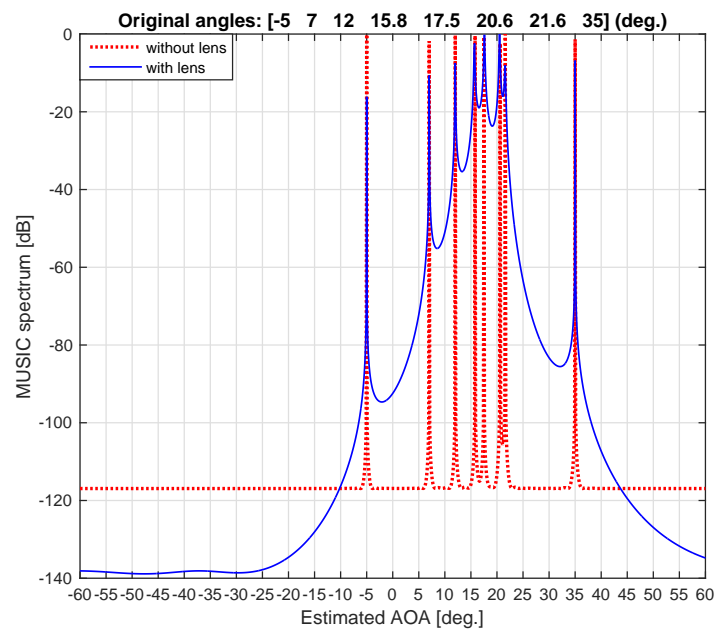

Fig. 7. Estimated MUSIC spectrum with lens versus without lens.
As an example, we show the MUSIC spectrum estimated for the original transmitted signals angles in Fig. 7 with lens versus without lens. It can be observed that the desired peaks (estimated DoAs) of the received signals are achieved at the same locations as in the without EM lens case, despite the reduced RF chains and the associated signal processing complexity.

\subsubsection{ESPRIT Algorithm}

Unlike MUSIC, the ESPRIT algorithm does not require an exhaustive search of all possible steering vectors [25]. The actual goal of this algorithm is to determine the rotation operator $\Phi$. The rotation operator $\Phi$ is actually a diagonal matrix of order $\boldsymbol{N} \times \boldsymbol{N}$ whose diagonal elements represent the phase delays between the sub-arrays doublets for the $N$ signals and is defined as

$$
\Phi=\operatorname{diag}\left[\mathrm{e}^{\mathrm{j} \psi_{1}}, \mathrm{e}^{\mathrm{j} \psi_{2}}, \mathrm{e}^{\mathrm{j} \psi_{3}}, \ldots, \mathrm{e}^{\mathrm{j} \psi_{N}}\right]
$$

where, $\psi=-2 k d_{x} \sin (\theta)$. Furthermore, by performing the EVD procedure on the covariance matrices of the received signals sub-arrays, two signal subspaces $E_{s 1}$ and $E_{s 2}$, are obtained. In this way, the rotation operator can be easily found and hence, the AoA of the $n^{\text {th }}$ signal source or the user terminal corresponding to the $n^{\text {th }}$ eigenvalue $\left(\lambda_{n}\right)$ of $\Phi$ can be estimated as [25]

$$
\theta_{n}=\sin ^{-1}\left(\frac{\arg \left(\lambda_{n}\right)}{k d_{x}}\right)
$$

In Fig. 8, we show the estimated DoAs for the original transmitted signals angles using the ESPRIT algorithm with lens versus without lens. It can be observed that the desired results can be achieved with the proposed method which are comparable to the scenario without EM lens.

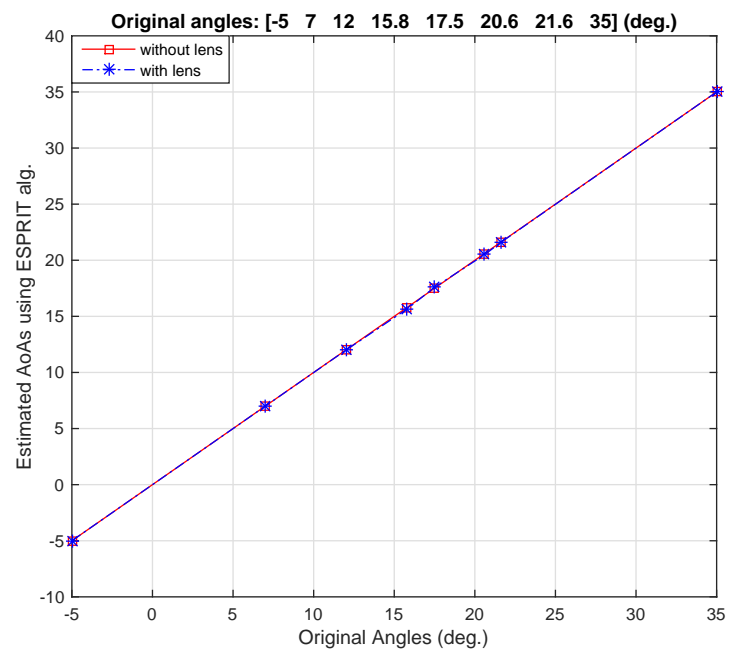

Fig. 8. Estimated DoAs/AoAs with lens versus without lens using the ESPRIT algorithm. 


\subsubsection{Root-MUSIC Algorithm}

The root-MUSIC is a variant of the ordinary MUSIC algorithm which starts with the MUSIC spectrum as defined in (12). It is applied to the ULA which generally provides better DoA estimation results than the traditional MUSIC [25]. In this method, after calculating the MUSIC spectrum, roots of a polynomial are found. Roots which are very close to the unit circle, provide the DoA estimation [21]. Let us assume $\mathbf{Q}=\mathbf{E}_{N} \mathbf{E}_{N}^{\mathrm{H}}$ then the MUSIC spectrum expression becomes

$$
\mathbf{p}_{\text {MUSIC }}(\theta)=\frac{1}{\left|\mathbf{v}^{H}(\theta) \mathbf{Q v}(\theta)\right|} .
$$

As mentioned earlier, the $m^{\text {th }}$ antenna element of the steering vector $v_{m}(\theta)$ is denoted as $v_{m}(\theta)=\mathrm{e}^{-\mathrm{j} m k d \sin (\theta)}$ then the denominator of (15) can be rewritten and simplified in a polynomial expression $U(x)$ as $[21,25]$

$$
U(x)=\sum_{p=-M+1}^{M-1} Q_{p} x^{-1} .
$$

In this way, the polynomial $U(x)$ is resolved on a unit circle where the spectrum peaks are equated to the roots of the $U(x)$ which are close to the unit circle $\left(\left|x_{n} \simeq 1\right|\right)$ so that the $n^{\text {th }}$ pole of the $U(x)$, denoting to the $n^{\text {th }}$ user signal, at $x_{n}=\left|x_{n}\right| \mathrm{e}^{\mathrm{j} \arg \left(x_{n}\right)}$ will give the AoA estimation as [21]

$$
\theta_{n}=\sin ^{-1}\left(\frac{\arg \left(x_{n}\right)}{k d}\right) \text {. }
$$

As an example, in Fig. 9 we show the estimated DoAs for the original transmitted signals angles using the Root-MUSIC algorithm with lens versus without lens. The obtained results indicate that the proposed method, using the EM lens, provides similar desired results as those obtained without lens. It is noted that by using the subspace based algorithms in the EM lens based system in a situation where each source signal is focused on a different subset consisting of $J$ antennas out of $M(J \lll M)$, then, the maximum source signals that can be resolved at one time would be $N_{\text {lens }}=(M / J)(J-1)$.

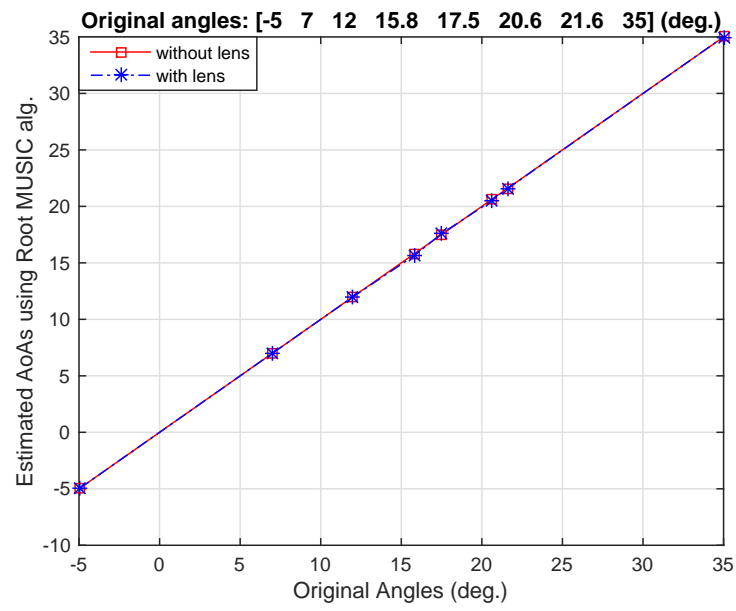

Fig. 9. Estimated DoAs/AoAs with lens versus without lens using the Root-MUSIC algorithm.
They are marginally less than the without EM lens case, i.e., $N_{\text {woLens }}=M-1$. Nevertheless, the performance of the EM lens for a number of source signals $N \leq N_{\text {lens }}$, despite reduced hardware and computational complexity, is slightly better than the without EM lens system. This is because the latter suffers from interference of multiple source signals on the antenna array while the former has focusing and additional interference rejection property as explained previously. Therefore, a tradeoff can be made between complexity and ability to resolve distinct DoAs.

\section{Simulation Results}

In this Sec., we provide the simulation results of the mean squared error (MSE) in degrees in order to investigate the performance of the proposed approach for the DoA estimation in the EM lens assisted massive antenna system. In this regard, it has been observed in the simulation process that all three subspace-based high resolution algorithms (MUSIC, ESPRIT and Root-MUSIC) perform very similarly to each other. Thus, for brevity, here we show the results of our proposed method with the root-MUSIC algorithm whose performance is slightly better than the other two. In this context, we consider a BS consisting of a ULA with $M=100$ antenna elements with elements spacing $d=\lambda / 2$. Due to the practical limitation of the antenna array angle coverage, we assume the DoA estimation range of the ULA as $-60^{\circ}$ to $+60^{\circ}$. Further, we consider $N=8$ user terminals and generate the corresponding random arrival angles, whose MSE performance is to be investigated. Furthermore, the lens power distribution function $a(\theta)$ is modeled by using (3) with peak power location $\bar{i}(\theta)=i_{\Delta+1}+\left(\left(\theta+60^{\circ}\right) /\left(2 \times 60^{\circ}\right)\right)\left(i_{M-\Delta}-i_{\Delta+1}\right)$ and variance $\sigma^{2}=(5 / 6 d)^{2}$. Note that the location of $m^{\text {th }}$ antenna element in terms of the wavelength $(\lambda)$ is defined as $i_{m}=(m-1) d-(M-1) d / 2$, where $m=1, \ldots, M$. We average all the estimated results over 10000 realizations of the randomly generated signals.

Having outlined our initial assumed parameters, we now discuss the performance results obtained from the proposed method in four different experiments and compare the results to the case without lens. In the initial three following experiments, we obtain the MSE performance results as a function of SNR (dB), snapshots and the number of antennas selected from the excited subsets, where, in general, the MSE expression is defined as [29]

$$
\mathrm{MSE}=\mathbb{E}\left[\left|\theta_{n}-\hat{\theta}_{n}\right|^{2}\right]
$$

where $\theta_{n}$ is the actual angle and $\hat{\theta}_{n}$ represents the estimated DoA of the $n^{\text {th }}$ user.

Experiment 1: In this part, we compute the MSE as a function of the SNR in $\mathrm{dB}$ by considering both scenarios (with lens and without lens) while we keep all other parameters constant. The MSE simulation graph obtained from this setup is provided in Fig. 10. The estimated MSE values with the EM lens are in good agreement with the MSE values with- 
out the EM lens, despite reduced hardware implementations and signal processing complexity. However, as expected, the performance of the proposed method looks slightly better as compared to without lens system. This is due to the fact that in the conventional system without EM lens, on average, the power received from each user signal is evenly distributed across all the antennas; therefore, for each user the desired signals at all the $\mathrm{M}$ receive antennas are equally corrupted by other user signals. As a result, this has the affects on the estimated DoAs resolution due to the interference of multiple source signals. In contrast, with the EM lens system as mentioned earlier, the received signals from different users are focused at different subsets of the receive antennas due to the AoA-dependent energy focusing provided by the EM lens. Consequently, this spatial interference rejection by the EM lens system provides a slightly less MSE in the DoA estimation than that without the EM lens system. Such spatial interference rejection can be achieved if $\min _{u \neq k}\left|m^{*}\left(\theta_{k}\right)-m^{*}\left(\theta_{u}\right)\right| \geq J$, where $m^{*}$ and $J$ represent the peak power location of an excited subset of antennas and the number of antennas in a subset, respectively. This is also true for the next Experiment 2.

Experiment 2: The MSE of both scenarios (with lens and without lens) has also been analyzed as a function of the number of signal snapshots (samples) taken. The results are shown in Fig. 11. It can be seen that the results obtained in the case with the lens are in good agreement to the case without the lens. Nevertheless, the performance gap between the two systems (with and without lens) still remains there, particularly for moderate values of snapshots, which shows the advantage of the EM lens focusing ability.

Experiment 3: In this experiment, we compute the MSE as a function of the number of selected antennas (RF-chains) $\hat{S}$ in a subset with fixed $\Delta=5(J=11)$ subset size, $\mathrm{SNR}=10 \mathrm{~dB}$ and snapshots $=200$, in the lens scenario. After running the simulation with this setup, the resulting MSE curve is shown in Fig. 12. It can be observed that if the number of selected antennas $\hat{S}$ in a subset is less than $J$, the performance starts to degrade. However, the performance improves if the number of selected antennas $\hat{S}$ is the same as the size of subset $J$. Hence, it can be remarked that with different values for the number of optimal antenna elements selected, our proposed method is capable of providing positive results.

Experiment 4: We further investigate the average received SNR performance for the two systems with lens and without lens. This is relevant to assess the performance of the system should it be used for data transmission as well. In this regard, we consider $M=100$ antennas, $\Delta=2$ and all other parameters/mathematical expressions as mentioned in the EM lens related work done in [3]. Figure 13 shows the average received SNR at the array output as a function of the training/transmission SNR ( $\rho_{\mathrm{tr}}$ - the ratio of the transmitted power to the noise power) for one randomly selected mobile receiver with its estimated/known AoA. It can be observed that the results obtained with lens look prominent over the without lens case particularly for $\rho_{\text {tr }}$ less than $10 \mathrm{~dB}$. This is due to the focusing and the additional spatial interference rejection ability of the EM lens. Thus, it shows that the proposed method can also be used for the data transfer application.

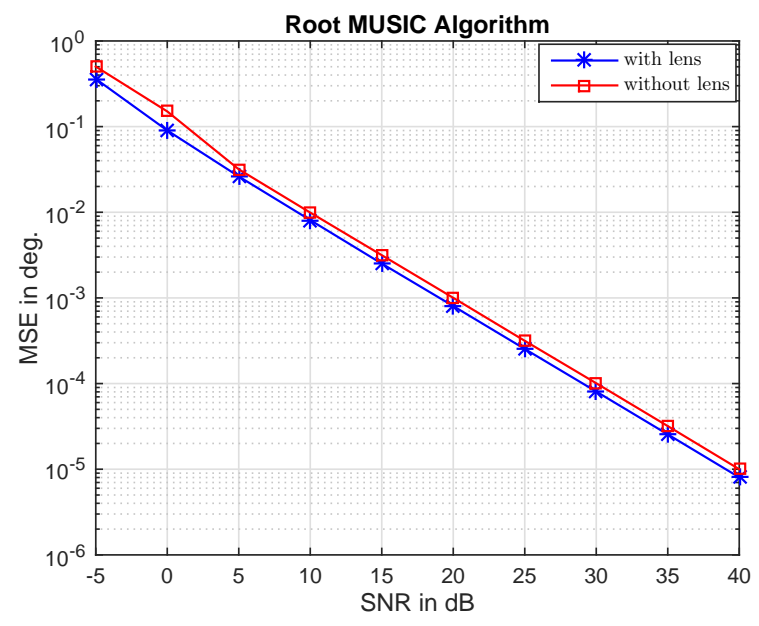

Fig. 10. MSE performance with lens versus without lens as a function of SNR $(\mathrm{dB})$.

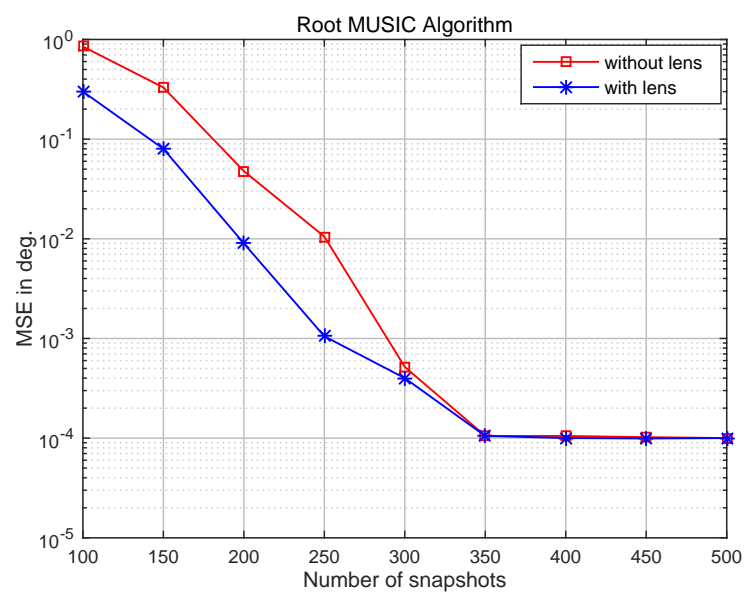

Fig. 11. MSE performance with lens versus without lens for different values of snapshots.

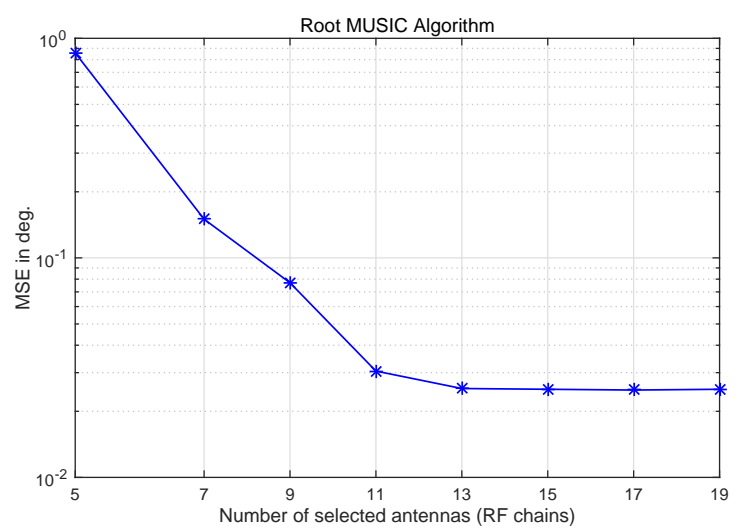

Fig. 12. MSE performance with lens as a function of number of antennas selected in a subset. 


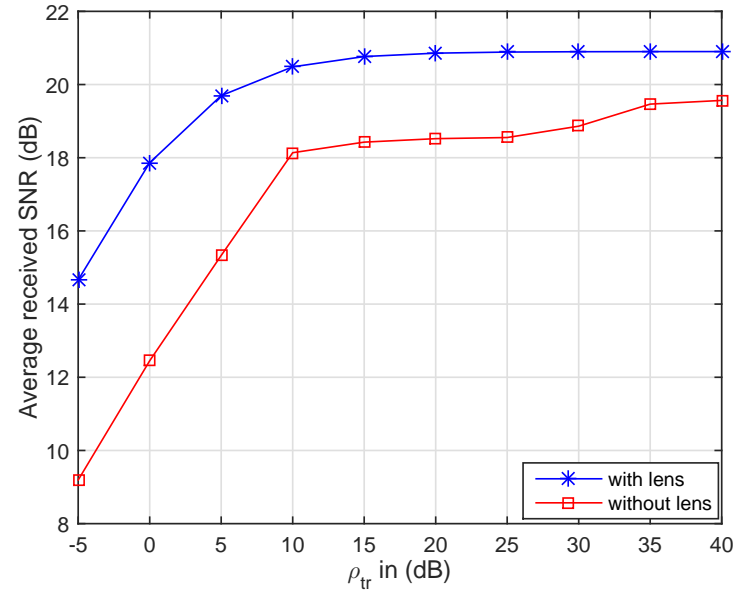

Fig. 13. Performance of average received SNR at the array output as a function of $\rho_{\text {tr }}$ for the two systems with lens and without lens.

\section{Conclusion and Future Work}

\subsection{Conclusion}

By combining the EM lens with a massive antenna system, this paper presents an approach for the LoS-DoA estimation using a subsets-based antenna selection method and the subspace-based high resolution algorithms. The EM lens has the ability to vary the path of the received signal as a function of the incident angle in order to focus energy on a smaller number of antenna elements (called subset). This focusing property of the EM lens provides a great opportunity to estimate the DoA of the user terminals precisely with reduced hardware (RF chains) and number of signals to be processed. Furthermore, the MSE performance of both scenarios (i.e. the technique with the lens and the conventional technique without the lens) has been evaluated in combination with subspace-based DoA estimation algorithms. The simulation results obtained have shown that the EM lens-focusing antenna approach is capable of providing positive DoA estimation results in the massive antenna system. Consequently, the results are promising and encourage further studies for potential applications in next generation networks such as $5 \mathrm{G}$.

\subsection{Future Work}

Even though the key elements the Lens based DoA estimation have been addressed in this paper, there are numerous future work directions as mentioned below.

- 2D/3D Array and DoA Configurations: As a proof of concept ULA has been considered in this work. The proposed approach can be further extended to design the $2 \mathrm{D} / 3 \mathrm{D}$ array in order to estimate the DoA in $2 \mathrm{D} / 3 \mathrm{D}$ space by applying the corresponding channel models and the appropriate 2D/3D DoA estimation techniques for instance 2D-MUSIC.
- DoA Estimation in Multipath Propagation: Since the EM lens separates and focuses each arrival path on different subsets of the massive antenna array as a function of the incident angle, by applying the joint angle and delay estimation (JADE) algorithms i.e., JADEMUSIC, AoA of each multipath can be estimated. Then, the first arrival path's (FAP) angle can be used as a estimated DoA of the received signal by assuming that FAP is the LoS component.

- DoA Estimation in Lens based Millimeter Wave Communications: Generally, massive antenna arrays are recommended to be used at the transmitter and/or receiver of the millimeter wave (mmWave) systems to achieve significant beamforming gains and to compensate the severe path loss. Thus, the concept of EM lens enabled massive antenna can be extended to the mmWave system that has great potential for the next generation advanced wireless networks i.e., 5G.

- DoA Estimation in RF-domain: In order to reduce the complexity of the standard DoA algorithms, it would be interesting to explore the DoA estimation in $\mathrm{RF} / \mathrm{Analog}$ domain such as sum-difference patterns based DoA estimation using the $180^{\circ}$ hybrid rat race ring couplers together with EM lens [30].

\section{Acknowledgments}

The authors wish to acknowledge the Higher Education Commission (HEC) of Pakistan and OeAD, Austria for the financial support of S. Shaikh to carry out the research work at the University of Klagenfurt, Austria.

\section{References}

[1] LARSSON, E., EDFORS, O., TUFVESSON, F., et al. Massive MIMO for next generation wireless systems. IEEE Communication Magazine, 2014, vol. 52, no. 2, p. 186-195. DOI: 10.1109/MCOM.2014.6736761

[2] JO, K., KO, Y., PARK, C., et al. Multiple-antenna post low noise amplifier RF combining. In Proceedings of the IEEE International Conference on Global Communications (GLOBECOM). Honolulu (Hawaii), 2009, p. 1-5. DOI: 10.1109/GLOCOMW.2009.5360684

[3] ZENG, Y., ZHANG, R., CHEN, Z. Electromagnetic lens-focusing antenna enabled massive MIMO: Performance improvement and cost reduction. IEEE Journal on Selected Areas in Communications, 2014, vol. 32, no. 6, p. 1194-1206. DOI: 10.1109/JSAC.2014.2328151

[4] GAO, X., EDFORS, O., TUFVESSON, F.,et al. Massive MIMO in real propagation environments: Do all antennas contribute equally? IEEE Transactions on Communications, 2015, vol. 63, no. 11, p. 3917-3928. DOI: 10.1109/TCOMM.2015.2462350

[5] SHAIKH, S., TONELLO, A. Localization based on angle of arrival in EM lens-focusing massive MIMO. In Proceedings of the IEEE International Conference on Consumer Electronics (ICCE). Berlin (Germany), 2016, p. 127-131. DOI: 10.1109/ICCE-Berlin.2016.7684736

[6] SAVIC, V., LARSSON, E. Fingerprinting-based positioning in distributed massive MIMO systems. In Proceedings of the IEEE International Conference on Vehicular Technology (VTC Fall). Boston (USA), 2015, p. 1-5. DOI: 10.1109/VTCFall.2015.7390953 
[7] TONELLO, A., INSERRA, D. Radio positioning based on DoA estimation: an implementation perspective. In Proceedings of the IEEE International Conference on Communications (ICC). Budapest (Hungary), 2013, p. 27-31. DOI: 10.1109/ICCW.2013.6649195

[8] SHAIKH, S., TEKIN, I. Two axis direction finding antenna system using sum-difference patterns in X-band. Microwave and $O p$ tical Technology Letters, 2015, vol. 57, no. 9, p. 2085-2092. DOI: 10.1002/mop.29269

[9] CHEN, H., WAN, Q., FAN, R., et al. Direction-of-arrival estimation based on sparse recovery with second-order statistics. Radioengineering, 2015, vol. 24, no. 1, p. 208-213. DOI: 10.13164/re.2015.0208

[10] SUN, L., WANG, H., XU, G. An efficient sparse representation algorithm for direction-of-arrival estimation. Radioengineering, 2013, vol. 22 , no. 3, p. 834-840. ISSN: $1805-9600$

[11] PIKSA, P., ZVANOVEC, S., CERNY, P. Elliptic and hyperbolic dielectric lens antennas in mm-waves. Radioengineering, 2011, vol. 20, no. 1, p. 270-275. DOI: 10.13164/re. 2011.

[12] SHAFIN, R., LIU, L., ZHANG, J. DoA estimation and RMSE characterization for 3D massive-MIMO/FD-MIMO OFDM system. In Proceedings of the IEEE International Conference on Global Communications (GLOBECOM). San Diego (USA), 2015, p. 1-6. DOI: 10.1109/TWC.2016.2594173

[13] WANG A., LIU, L., ZHANG, J. Low Complexity direction of arrival (DoA) estimation for 2D massive MIMO systems. In Proceedings of the IEEE International Conference on Global Communications (GLOBECOM). Anaheim (USA), 2012, p. 703-707. DOI: 10.1109/GLOCOMW.2012.6477660

[14] LUTZ, S., WALTER, T. Lens based 77 GHz TDM MIMO radar sensor for angular estimation in multitarget environments. In Proceedings of the IEEE International Conference on European Radar (EuRAD). Nuremberg (Germany), 2013, p. 212-215. ISBN: 978-2-87487-033-0

[15] LEVINE, E., MALAMUD, G., SHTRIKMAN, S., et al. A study of microstrip array antennas with the feed network. IEEE Transactions on Antennas and Propagation, 1989, vol. 37, no. 4, p. 426-434. DOI: $10.1109 / 8.24162$

[16] ANTENNA ARRAYS (PHASED ARRAYS). [Online] Cited 2017-02-24. Available at: http://www.antennatheory.com/arrays/main.php/phased

[17] BALANIS, C. Antenna Theory, Analysis and Design. 3rd ed., rev. New Jersey (USA): John Wiley and Sons Inc, 2005. ISBN: 0-471-66782-X

[18] STUTZMAN, W., THIELE, G. Antenna Theory and Design. 2nd ed., rev. New York (USA): John Wiley and Sons Inc, 1998. ISBN: 0-471-02590-9

[19] ZENG, Y., ZHANG, R. Millimeter wave MIMO with lens antenna array: a new path division multiplexing paradigm. IEEE Transactions on Communications, 2016, vol. 64, no. 4, p. 1557-1571. DOI: 10.1109/TCOMM.2016.2533490

[20] LAU, P. CHEN, Z., QING, X. Electromagnetic field distribution of lens antennas. In Proceedings of the Asia-Pacific Conference on Antennas Propagation, 2013, p. 1-2.

[21] WAWERU, N., KONDITI, O., LANGAT, P. Performance analysis MUSIC, Root-MUSIC and ESPRIT DOA estimation algorithm. International Journal of Electrical, Computer, Energetic, Electronic and Communication Engineering, 2014, vol. 8, no. 1, p. 209-216.

[22] ADAM, I., ISLAM, M. Performance study of direction of arrival (DOA) estimation algorithms for linear array antenna. In Proceedings of the International Conference on Signal Processing Systems. Singapore, 2009, p. 268-271. DOI: 10.1109/ICSPS.2009.47

[23] GROSS, F. Smart Antennas for Wireless Communications with Matlab. New York (USA): McGraw Hill, 2005. ISBN: 978-0071447898
[24] TEHRANI, M., LAURIN, J., SAVARIA, Y. Multiple targets directionof-arrival estimation in frequency scanning array antennas. IET Radar, Sonar and Navigation, 2016, vol. 10, no. 3, p. 624-631. DOI: 10.1049/iet-rsn.2015.0401

[25] ALLEN, B., GHAVAMI, M. Adaptive Array Systems. 1st ed., rev. England: John Wiley and Sons Inc, 2005. ISBN: 978-0-470-86189-9

[26] SANAVEI, S., NOSRATINIA A. Antenna selection in MIMO systems. IEEE Communication Magazine, 2004, vol. 42, no. 10 DOI: 10.1109/MCOM.2004.1341263

[27] LIU, A., LAU,V. Phase only RF precoding for massive MIMO systems with limited RF chains. IEEE Transactions on Signal Processing, 2014, vol. 62, no. 17, p. 4505-4515. DOI: $10.1109 /$ TSP.2014.2337840

[28] AL-SHURAIFI, M., AL-RAWESHIDY, H. Optimizing antenna selection using limited CSI for massive MIMO systems. In Proceedings of the Int. Conference on Innovative Computing Technology (INTECH). Luton (UK), 2014, p. 180-184. DOI: 10.1109/INTECH.2014.6927740

[29] MA, Y., CAI, J., BAO, D., et al. An efficient MUSIC algorithm using subspace projection. In Proceedings of the IEEE International Conference on Signal Processing, Communications and Computing (ICSPCC). Ningbo (China), 2015, p. 1-6. DOI: 10.1109/ICSPCC.2015.7338949

[30] SHAIKH, S., TONELLO, A. Performance Analysis of $180^{\circ}$ HRR Coupler Used for Direction Finding with an Antenna Array. International Journal of Online Engineering (iJOE), 2017. [To be appeared]

\section{About the Authors...}

Sarmad Ahmed SHAIKH was born in Pakistan. He received the BS Telecommunication Engineering degree from FAST-NUCES, Pakistan in 2011, and the M.Sc. Electronics Engineering degree from Sabanci University, Istanbul, Turkey in 2015. Currently, he is working towards the $\mathrm{PhD}$ degree at the Institute of Networked and Embedded Systems, University of Klagenfurt, Klagenfurt, Austria. His research interests include $\mathrm{RF} /$ wireless communications, localization of source signals and antenna designing.

Andrea M. TONELLO was born in Italy. He received the laurea degree in electrical engineering (Hons.) and the Ph.D in electronics and telecommunications from the University of Padova, Padova, Italy, in 1996 and in 2003, respectively. From 1997 to 2002, he was with Bell Labs-Lucent Technologies, Whippany, NJ, USA, first as a Member of the Technical Staff. Then, he was promoted to Technical Manager and appointed to Managing Director of the Bell Labs Italy division. In 2003, he joined the University of Udine, Udine, Italy, where he became Aggregate Professor in 2005 and Associate Professor in 2014. Currently, he is the Chair of the Embedded Communication Systems Group at the University of Klagenfurt, Klagenfurt, Austria. Dr. Tonello received several awards, including the Distinguished Visiting Fellowship from the Royal Academy of Engineering, U.K., in 2010, the IEEE VTS Distinguished Lecturer Award in 2011-2015, and eight best paper awards. He is the Chair of the IEEE Communications Society Technical Committee on Power Line Communications and Associate Editor of IEEE Transactions on Communications and IEEE Access. 